\title{
A CLASSIFICATION OF IMMERSED KNOTS
}

\section{MAX K. AGOSTON}

This paper in a sense completes the study begun in [6] and [3]. We shall make extensive use of the notation and results of [6] and shall show that the group of immersed homotopy $n$-spheres in $m$-space fits naturally into exact sequences similar to those of [6]. Alternatively, one can look on this paper as giving geometric meaning to the groups $\pi_{n} G$ and $\pi_{n}\left(G, S O_{q}\right)$. (See also [4]. For additional information about immersions see $\$ 4$ of [3].) In $\$ 1$ we give the necessary definitions; the main results are stated in $\S 2$. We would like to thank the referee for some useful observations.

1. Preliminaries. Throughout this paper all manifolds will be $C^{\infty}$, compact, and oriented. All maps are transverse to any boundaries and take boundaries to boundaries. If $f: M^{n} \rightarrow W^{m}$ is an immersion, we orient the normal bundle of $f, \nu_{f}$, by the equation

$$
\tau_{M}+v_{f}=f^{*} \tau_{W},
$$

where $\tau_{M}, \tau_{W}$ are the tangent bundles of $M, W$, respectively. The boundary of $M, \partial M$, is oriented by the equation

$$
\xi+\tau_{\partial M}=\tau_{M} \mid \partial M
$$

where $\xi$ is a line bundle of vectors orthogonal to $\partial M$ with the vectors pointing out from $M$ oriented positively. $-M$ denotes $M$ with the negative orientation. Given a vector bundle $\eta$ over $M$ we shall always identify $M$ with the zero-section of $\eta$; also, we do not distinguish between the normal disk bundle of a submanifold and a tubular neighborhood.

Next, we say that $(f, \mathcal{F}): M^{n} \rightarrow W^{n}$ is a framed immersion if $f: M \rightarrow W$ is an immersion and $\mathcal{F}$ is a framing of $\nu_{f}$, i.e., $\mathcal{F}=\left(f_{1}, \cdots, f_{m-n}\right)$ is an ordered collection of orthogonal vector fields of $\nu_{f}$ (this is compatible with [6] in case $f$ is an imbedding). Now let $M^{n}$ and $W^{m}$ be closed manifolds (boundaries are excluded only for simplicity). Two immersions $f, g: M \rightarrow W$ are $h$-cobordant if there is an $h$-cobordism $V^{n+1}$ with $\partial V=M \cup-M$ and an immersion $H: V \rightarrow W \times[0,1]$ with $H \mid M=f \times 0$ and $H \mid-M=g \times 1$. Two framed immersions $(f, \mathcal{F})$, $(g, \mathcal{G}): M \rightarrow W$ are $h$-cobordant if there is an $h$-cobordism $V^{n+1}$ with $\partial V=M \cup-M$ and a framed immersion $(H, \mathfrak{F}): V \rightarrow W \times[0,1]$ with $(H, \mathcal{F}) \mid M=(f, \mathcal{F}) \times 0$ and $(H, \mathcal{F}) \mid-M=(g, \mathcal{S}) \times 1$. We use $[M, f]$, $[M, f, \mathcal{F}]$ to denote the $h$-cobordism class of $f,(f, \mathscr{F})$, respectively-

Received by the editors May 28, 1969. 
the range $W$ will always be clear from the context. Also, let us recall the notion of regular homotopy between immersions (see [5]). Two framed immersions $(f, \mathcal{F}),(g, \mathcal{G}): M \rightarrow W$ will be called regularly homotopic if there is a 1-parameter family of framed immersions $\left(H_{t}, \mathfrak{F}_{t}\right): M \rightarrow W$ with $\left(H_{0}, \mathfrak{F}_{0}\right)=(f, \mathfrak{F})$ and $\left(H_{1}, \mathfrak{F}_{1}\right)=(g, \mathcal{S})$.

As usual, $D^{n}$ will be the closed unit ball in Euclidean $n$-space $R^{n}$ with the natural orientation and $S^{n-1}=\partial D^{n}$. $G_{n}$ denotes the $H$-space of maps of $S^{n-1} \rightarrow S^{n-1}$ of degree +1 and $S O_{n}$ will be the subspace of orthogonal maps. The natural inclusion $R^{n} \subseteq R^{n+1}$ gives rise to inclusions $D^{n} \subseteq D^{n+1}, \quad G_{n} \subseteq G_{n+1}, \quad S O_{n} \subseteq S O_{n+1}$. Let $G=\lim _{n \rightarrow \infty} G_{n}$ and $S O=\lim _{n \rightarrow \infty} S O_{n}$.

2. The exact sequences. For the remainder of this paper we assume that $n \geqq 5$ and $k=m-n \geqq 3$. We define $I^{m, n}$ to be the set of $h$-cobordism classes of immersed homotopy $n$-spheres in $S^{m}$ and $I_{f}^{m, n}$ to be the set of $h$-cobordism classes of framed immersed homotopy $n$-spheres in $S^{m}$. There is an obvious operation of connected sum which makes $I^{m, n}$ and $I_{f}^{m, n}$ into abelian groups (see [2, \$1.3 and \$1.4]). Using [7] it is easy to see that $\left[\Sigma^{n}, f\right]=0 \in I^{m, n}$ (or $\left[\Sigma^{n}, f, \mathcal{F}\right]=0 \in I_{f}^{m, n}$ ) if and only if $\Sigma^{n}$ is the boundary of an $(n+1)$-disk $U^{n+1}$ and there is an immersion $H: U^{n+1} \rightarrow D^{m+1}$ (or framed immersion $(H, \mathfrak{F}): U^{n+1} \rightarrow D^{m+1}$ ) so that $H \mid \Sigma=f$ (or $(H, \mathfrak{F}) \mid \Sigma=(f, \mathcal{F})$ ).

Define groups $P_{n}$ as follows:

$$
\begin{aligned}
P_{n} & =Z, & & n \equiv 0(\bmod 4) \\
& =Z_{2}, & & n \equiv 2(\bmod 4) \\
& =0, & & n \text { odd } .
\end{aligned}
$$

In $\S 3$ we shall define homomorphisms $\bar{\omega}_{i}, \bar{\phi}_{i}$, and $\bar{\partial}_{i}$ making the following sequences exact:

$(1)_{k} \quad \cdots \rightarrow \pi_{n} S \mathrm{O}_{k} \stackrel{\bar{\omega}_{1}}{\rightarrow} I_{f}^{m, n} \stackrel{\bar{\phi}_{1}}{\rightarrow} I^{m, n} \stackrel{\bar{\partial}_{1}}{\rightarrow} \pi_{n-1} S O_{k} \stackrel{\bar{\omega}_{1}}{\rightarrow} I_{f}^{m-1, n-1} \rightarrow \cdots$

$$
\begin{aligned}
& \ldots \rightarrow I_{f}^{m, n} \stackrel{\bar{\omega}_{2}}{\rightarrow} \pi_{n} G \stackrel{\bar{\phi}_{2}}{\rightarrow} P_{n} \stackrel{\bar{\partial}_{2}}{\rightarrow} I_{f}^{m-1, n-1} \stackrel{\bar{\omega}_{2}}{\rightarrow} \pi_{n-1} G \rightarrow \cdots \\
& \ldots \rightarrow I^{m, n} \stackrel{\bar{\omega}_{3}}{\rightarrow} \pi_{n}\left(G, S O_{k}\right) \stackrel{\bar{\phi}_{3}}{\rightarrow} P_{n} \stackrel{\bar{\partial}_{3}}{\rightarrow} I^{m-1, n-1} \stackrel{\bar{\omega}_{3}}{\rightarrow} \pi_{n-1}\left(G, S O_{k}\right) \rightarrow \cdots .
\end{aligned}
$$

We also get a commutative (up to sign) diagram:

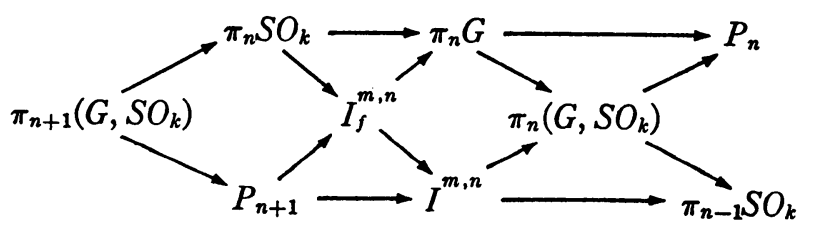


Next, let $\operatorname{Im}^{m, n}\left(I m_{f}^{m, n}\right)$ be the group of regular homotopy classes of (framed) immersions of $S^{n}$ in $S^{m}$. The group operation is again the connected sum in both cases. Then it follows from [5] that there are natural isomorphisms $I m^{m, n} \approx \pi_{n} V_{m, n} \approx \pi_{n}\left(S O, S O_{k}\right)$ and $I m_{f}^{m, n}$ $\approx \pi_{n} S O_{m} \approx \pi_{n} S O$. Finally, we also consider the groups $C_{n}^{k}$ and $F C_{n}^{k}$ of isotopy classes of imbedding, respectively, framed imbeddings, of $S^{n}$ in $S^{m}$ and the groups $\theta^{m, n}$ and $\theta_{f}^{m, n}$ of $h$-cobordism classes of imbedded, respectively, framed imbedded, homotopy $n$-spheres in $S^{m}$. Collecting the results of [3], [6], and this paper we get a great many interrelated exact sequences which we shall not bother to write out here. In addition, there are natural suspension maps of the sequences $(l)_{k} \stackrel{s}{\rightarrow}(l)_{k+N}, l=1,2,3$ and $N \geqq 0$, where we take the rear extensions of framings as described in $[6, \S 1.2]$.

We shall display two interesting diagrams of exact sequences which are derived from standard diagram chasing:

$(5)_{k}$

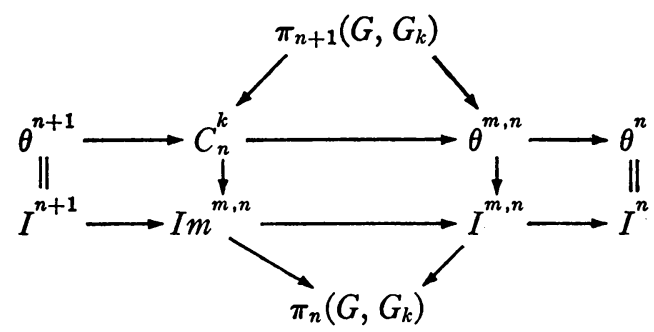

$(6)_{k} \quad \pi_{n} S^{k}=\left.\left.\pi_{n}\left(S O_{k+1}, S O_{k}\right)\right|_{I m^{m, n} \longrightarrow I m^{m+1, n}}\right|_{n-1}\left(S O_{k+1}, S O_{k}\right)=\pi_{n-1} S^{k}$,

where $\theta^{n}=\theta^{m+N, n}$ and $I^{n}=I^{m+N, n}$ for $N>n$. Also, observe that $I_{f}^{m, n}$ is independent of $m$.

In conclusion, we point out that this paper could have been extended to the case of "relative" immersed knots à la [1].

3. The maps $\bar{\omega}_{i}, \bar{\phi}_{i}, \bar{\partial}_{i}$. In this section we shall define the maps $\bar{\omega}_{i}, \bar{\phi}_{i}$, and $\bar{\partial}_{i}$. Let $\omega_{i}, \phi_{i}$, and $\partial_{i}$ be defined as in [6] and let $j_{1}: \theta^{m, n} \rightarrow I^{m, n}$, $j_{2}: \theta_{f}^{m, n} \rightarrow I_{f}^{m, n}$ be the obvious maps which assign to each $h$-cobordism class of imbeddings, respectively, framed imbeddings, its corresponding $h$-cobordism class as an immersion, respectively, framed immer- 
sion. (It should be clear that $\theta^{m, n}$ and $\theta_{f}^{m, n}$ can be so interpreted. See also [1].)

Define

$$
\begin{aligned}
& \bar{\omega}_{1}=j_{2} \omega_{1}, \quad \bar{\phi}_{2}=\phi_{2}, \\
& \bar{\phi}_{3}=\text { composition of } \pi_{n}\left(G, S O_{k}\right) \rightarrow \pi_{n}(G, S O) \stackrel{\phi_{3}}{\rightarrow} P_{n}, \\
& \bar{\partial}_{2}=j_{2} \partial_{2}, \quad \bar{\partial}_{3}=j_{1} \partial_{3} .
\end{aligned}
$$

Let $\left[\Sigma^{n}, f, \mathcal{F}\right] \in I_{f}^{m, n}$ and $\left[N^{n}, g\right] \in I^{m, n}$. Define

$$
\begin{gathered}
\bar{\phi}_{1}\left(\left[\Sigma^{n}, f, \mathfrak{F}\right]\right)=\left[\Sigma^{n}, f\right] \in I^{m, n}, \\
\bar{\partial}_{1}\left(\left[N^{n}, g\right]\right)=\nu_{g} \in \pi_{n-1} S O_{k},
\end{gathered}
$$

where we identify the normal bundle $\nu_{g}$ with the element in $\pi_{n-1} S O_{k}$ that it determines by the classification theorem of bundles over spheres.

Finally we come to the maps $\bar{\omega}_{2}$ and $\bar{\omega}_{3}$ which, together with the exactness of $(2)_{k}$ at $\pi_{n} G$ and $(3)_{k}$ at $\pi_{n}\left(G, S O_{k}\right)$, are really the heart of this paper.

LEMMA 3.1. $\theta_{f}^{N, n} \stackrel{j_{2}}{\approx} I_{f}^{N, n}$, for $N \geqq 2 n+1$.

Proof. Now all homotopy $n$-spheres $\Sigma^{n}$ imbed in $S^{N}$ and all immersions $f: \Sigma \rightarrow S^{N}$ are regularly homotopic by [5]. A regular homotopy also carries along the framing. Therefore $j_{2}$ is onto. That $j_{2}$ is one-toone follows from the fact that we may approximate regular homotopies by imbeddings using Whitney's theorem.

Thus we can define

$$
\bar{\omega}_{2}=\text { composition of } I_{f}^{m, n} \stackrel{S}{\rightarrow} I_{f}^{N, n} \stackrel{-j_{2}^{-1}}{\rightarrow} \theta_{f}^{N, n} \stackrel{\omega_{2}}{\rightarrow} \pi_{n} G,
$$

where $N \geqq 2 n+1$.

Now let $\left[\Sigma^{n}, f\right] \in I^{m, n}$. It is well known (see [7]) that $\Sigma^{n}$ is obtained by glueing two $n$-disks $D_{0}$ and $D_{1}$ together via a diffeomorphism of their boundaries. We may then take framings $\mathfrak{F}_{i}$ of $\nu_{f} \mid D_{i}, i=0,1$, and let $\alpha_{i}: D_{i} \times D^{k} \rightarrow \nu_{f} \mid D_{i}$ be imbeddings satisfying $\alpha_{i}(x, 0)=x, x \in D_{i}$ and $d \alpha_{i}\left(\mathcal{E}_{i}\right)=\mathcal{F}_{i}$, where $\mathcal{E}_{i}$ is the pull-back by the projection $D_{i} \times D^{k}$ $\rightarrow D^{k}$ of a positive frame at $0 \in D^{k}$. We can arrange it so that $\alpha_{1}(x, y)$ $=\alpha_{0}(x, \mu(x) y), x \in \partial D_{0}, y \in D^{k}$, for some $\mu: \partial D_{0} \rightarrow S O_{k}$. (Compare [6, $\S 3.3]$.) We shall use the notation $\mathfrak{F}_{1}\left|\partial D_{0}=\mu \mathcal{F}_{0}\right| \partial D_{0}$ to describe this situation. Now assume that $f \mid D_{0}$ is an imbedding and that $f\left(\Sigma-D_{0}\right)$ does not meet $f\left(D_{0}\right)$. Let $f^{\prime}$ be the composition of $\Sigma \stackrel{f}{\rightarrow} S^{m} \rightarrow S^{m+N}$, for some $N>n$, and let $\mathcal{F}_{i}^{\prime}$ be the framing of $\nu_{f^{\prime}} \mid D_{i}$ which is the rear ex- 
tension of $\mathcal{F}_{i}$. Next, move $f^{\prime}$ into an imbedding $g: \Sigma \rightarrow S^{m+N}$ via a regular homotopy $h_{t}$ satisfying $h_{t} \mid D_{0}=f^{\prime}$ and $h_{t}\left(\Sigma-D_{0}\right) \cap h_{t}\left(D_{0}\right)=\varnothing$. $h_{t}$ carries along the framing $\mathcal{F}_{i}^{\prime}$, so that we get a framing $\mathcal{G}_{i}$ of $\nu_{\theta} \mid D_{i}$ with $\mathcal{G}_{1}\left|\partial D_{0}=\bar{\mu} \mathcal{G}_{0}\right| \partial D_{0}$, where $\bar{\mu}$ is the composition of $\partial D_{0} \stackrel{\mu}{\rightarrow} S O_{k}$ $\rightarrow S O_{k+N}$. If we apply the construction of $[6, \S 3.3]$, to $\left(\Sigma, g, \S_{i}\right)$, we get an element $[\lambda] \in \pi_{n}\left(G, S O_{k}\right)$, i.e., if we let $u: S^{m+N}-g(\Sigma) \rightarrow S^{k+N-1}$ be a homotopy inverse of $y \rightarrow \alpha_{0}\left(x_{0}, y\right), x_{0} \in D_{0}$ with $u \alpha_{0}(x, y)=y$ for all $x \in D_{0}$, then $\lambda: D_{1} \rightarrow G_{k+N}$ is given by $\lambda(x)(y)=u \alpha_{1}(x, y), x \in D_{1}$, $y \in S^{k+N-1}$. Define

$$
\bar{\omega}_{3}([\Sigma, f])=[\lambda] .
$$

This finishes the definitions of all the maps and it is easy to see, using [6], that they are well defined homomorphisms.

4. Exactness. The proof of exactness of $(1)_{k},(2)_{k}$, and $(3)_{k}$ is very similar to the corresponding proofs given in [6]. In general, the only difference is that here we have immersions instead of imbeddings. Anyone who understands [6] can easily make the appropriate translations. We shall, however, outline a proof of exactness in those places that differ from the corresponding ones in [6]. One essential difference is the fact that any abstract framed surgery can be realized ambiently. Another is that framed immersions of $n$-spheres in $S^{m}$ are regularly homotopic to immersions in $S^{n+1}$.

We first prove exactness at $\pi_{n} G$ in $(2)_{k}$. That $\phi_{2} \bar{\omega}_{2}=0$ follows from $[6, \S 5.5]$, and the definition of $\bar{\omega}_{2}$ and $\bar{\phi}_{2}$. Let $[g] \in \pi_{n} G, g: S^{n} \rightarrow G_{N}$ and suppose $\bar{\phi}_{2}([g])=0$. Define $\bar{g}: S^{n} \times S^{N-1} \rightarrow S^{N-1}$ by $\bar{g}(x, y)=g(x)(y)$, $x \in S^{n}, y \in S^{N-1}$, and let $\Sigma=\bar{g}^{-1}(e), e \in S^{N-1}$. We may assume that $\Sigma$ is a framed $n$-submanifold of $S^{n} \times S^{N-1} \subseteq S^{n+N}$. In fact, since $\Phi_{2}([g])=0$, we may further assume that $\Sigma$ is a homotopy sphere (see $[6, \S 4.7])$. By Theorem 6.4 of $[5], \Sigma$ is regularly homotopic to a framed immersion $(\Sigma, f)$ in $S^{m}$. Then $\bar{\omega}_{2}([\Sigma, f])=[g]$.

Next, let us consider exactness at $I_{f}^{m, n}$. There is no problem in showing that $\bar{\omega}_{2} \bar{\partial}_{2}=0$. Suppose $[\Sigma, f, \mathcal{F}] \in I_{f}^{m, n}$ and $\bar{\omega}_{2}([\Sigma, f, \mathcal{F}])=0$. It follows from the definition of $\bar{\omega}_{2}$ and the exactness of the KervaireMilnor sequence that there is a $\pi$-manifold $W$ and a framing of its stable normal bundle so that $\partial W=\Sigma$ and the framing restricted to $\Sigma$ is essentially a suspension of $\mathcal{F}$. But then we can use [5] to obtain a framed immersion $(g, \mathcal{G}): W \rightarrow S^{m}$ so that $g \mid \Sigma=f$ and $\mathcal{G} \mid \Sigma=\mathcal{F}$. Define $\gamma=\gamma(W, \mathcal{g}) \in P_{n+1}$ as in $\S 4.5$ of $[6]$. Then $\bar{\partial}_{2}(\gamma)=[\Sigma, f, \mathcal{F}]$, because using [5] we can allow in the definition of $\bar{\partial}_{2}$ not only framed imbeddings of $W$ but also framed immersions.

This finishes our discussion of the exactness of $(2)_{k}$. Alternatively, 
one could observe first that $I_{f}^{m, n} \approx I_{f}^{n} \approx \theta_{f}^{n}$ using [5], so that exactness follows from the exactness of the Kervaire-Milnor sequence.

The commutativity (up to sign) of $(4)_{k}$ is proved as in [6] and so by $[6, \S 5.3]$, the exactness of $(3)_{k}$ will be established once we show that $\bar{\phi}_{3} \bar{\omega}_{3}=0$. But consider

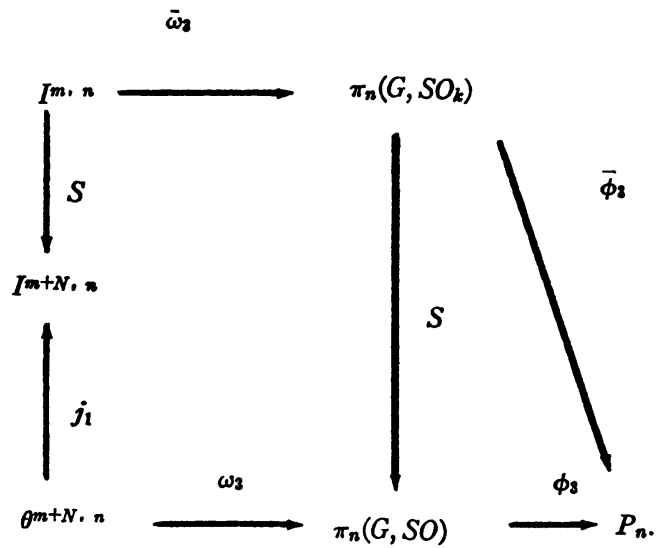

If $N>n$, then $j_{1}$ is an isomorphism (proved similarly to Lemma 3.1), and so $\phi_{3} \omega_{3}=\phi_{3} \omega_{3} j_{1}^{-1} S=0$.

\section{REFERENCES}

1. M. K. Agoston, Relative knots and tangential equivalences, Trans. Amer. Math. Soc. 137 (1969), 27-52.

2. A. Haefliger, Knotted (4k-1)-spheres in 6k-space, Ann. of Math. (2) 75 (1962), 452-466. MR $26 \# 3070$.

3. - Differentiable embeddings of $S^{n}$ in $S^{n+q}$ for $q>2$, Ann. of Math. (2) 83 (1966), 402-436. MR 34 \#2024.

4. - Lissage des immersions. I, Topology 6 (1967), 221-239. MR 34 \#8416.

5. M. W. Hirsch, Immersions of manifolds, Trans. Amer. Math. Soc. 93 (1959), 242-276. MR 22 \#9980.

6. J. Levine, A classification of differentiable knots, Ann. of Math. (2) 82 (1965), 15-50. MR 31 \#5211.

7. S. Smale, On the structure of manifolds, Amer. J. Math. 84 (1962), 387-399. MR 27 \#2991.

Wesleyan University, Middletown, Connecticut 06457 